\title{
Follow-through care for high-risk infants during the COVID-19 pandemic: lessons learned from the Vermont Oxford Network
}

\author{
Jonathan S. Litt (iD ${ }^{1,4}$, Charles E. Mercier (iD) $2,3,4$, Erika M. Edwards ${ }^{2,3}$, Kate Morrow ${ }^{3}$ and Roger Soll ${ }^{2,3}$ \\ (c) The Author(s), under exclusive licence to Springer Nature America, Inc. 2021
}

OBJECTIVE: The COVID-19 pandemic has altered the delivery of follow-up care for high-risk infants. We performed an audit to characterize programmatic responses in a quality improvement network.

STUDY DESIGN: We audited 43 North American-based follow-up programs of the Vermont Oxford Network Extremely Low Birth Weight Follow-up Study Group in October, 2020. Our electronic survey included yes/no, agree/disagree, and free text response items.

RESULT: The response rate was $67.4 \%$. Most programs altered capacity and the timing, frequency, or content of clinical assessments. Most perceived practice changes compromised their ability to ascertain infants' medical and developmental needs. There was a rapid uptake of telemedicine services. Despite challenges with implementation, many endorsed improved connectedness with families.

CONCLUSION: Programs adapted rapidly to meet the needs of high-risk infants during the pandemic. Clinical operations, assessment procedures, and quality metrics will also need to evolve. Quality improvement study group collaboratives are wellpositioned to coordinate such work.

Journal of Perinatology (2021) 41:2625-2630; https://doi.org/10.1038/s41372-021-01158-8

\section{INTRODUCTION}

The COVID-19 pandemic has disrupted the delivery of healthcare services in the United States. Health systems have been forced to invest their resources in helping those with critical needs due to coronavirus infections, diverting them away from services deemed non-urgent or non-essential [1]. As well, care management of chronic illnesses has been delayed or deferred due to stay-athome orders or fear of infection [2]. Despite lower rates of COVID19 in pediatric populations compared to adults [3], and even lower rates of severe disease requiring hospitalization [4, 5], shifts in resource allocation and deferral of health care maintenance have had a large negative impact on pediatric primary and subspecialty care [6]. This may be most consequential for children with medical and developmental complexity, given their high rates of needs and time-sensitive nature of developmental interventions [7].

Premature extremely low birth weight (ELBW) infants are uniquely vulnerable to having ongoing chronic health problems and developmental delays during childhood, and increased needs for community support services compared to term-born peers [8]. Managing and coordinating medical, therapeutic, and educational services are challenging for providers and families and accentuated by the pandemic. Parents report experiencing increased stress, anxiety, and isolation due to lockdowns [9]. Reduced availability of home-based clinical supports (e.g. visiting nurses, early intervention programs) [10] and non-clinical support systems (e.g. extended family and parent groups), causes additional strain and have limited resources available to young children and their families.

High-risk infant follow-up programs (HRIF) routinely provide medical and developmental surveillance and support to ELBW infants and their families. These clinics help coordinate and manage complex care among all services and members of the care team [10-12]. Challenges imposed by the coronavirus pandemic to HRIF operations include reduced subspecialist availability for elective, non-urgent services, and reluctance of parents to seek care for fear of their child's health, or their own [13]. Furthermore, assessing growth, lung function, neurologic status, and developmental skills requires a hands-on approach, made difficult by the use of cumbersome personal protective equipment and virtual telehealth visits.

The Vermont Oxford Network (VON) ELBW Infant Follow-up Study Group is a voluntary group of follow-up clinics collaborating to benchmark ELBW clinical outcomes through two years corrected age and improve both follow-up care and the value of follow-up services for ELBW infants and their families. Recognizing the opportunity and need to quickly adapt follow-up service delivery to restrictions imposed by the pandemic, we performed an audit to characterize programmatic responses to the pandemic among VON Follow-up Study Group members. We then undertook a qualitative analysis of free-text narrative responses to evaluate the perceived effect of practice changes on care delivery and quality, identify perceived barriers in providing services during the

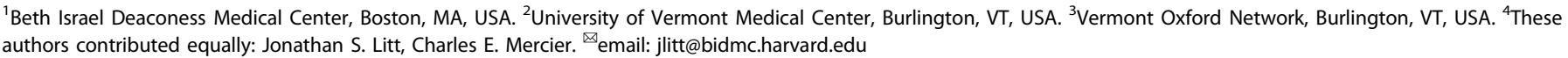

Received: 11 December 2020 Revised: 7 June 2021 Accepted: 14 June 2021

Published online: 27 July 2021 
pandemic, and explore the perceived impact of newly-adopted telemedicine platforms on high-risk infant follow-up.

\section{METHODS}

This project was a prospective, time-limited audit of follow-up care practices. Eligible participants were the 43 U.S.- and Canada-based HRIF programs belonging to the VON ELBW Infant Follow-up Study Group. All VON member centers with high-risk infant follow-up programs that provide medical and developmental support to infants after NICU discharge may elect to join the ELBW Infant Follow-up Study Group. The aim of the study group is to track the care needs, health services utilization, and neurodevelopmental outcomes of infants born $<27$ weeks' gestation or $\leq 1000$ grams at $18-24$ months of age corrected for gestation. VON oversees data collection and management and provides annual reports for each birth year cohort to be used for quality local improvement initiatives. We asked representatives from each program to work together and submit one response to the audit survey. This activity did not meet the criteria for human subjects research. All participating member institutions have active data-sharing agreements with VON.

The audit survey instrument was developed by the project leadership team and included 16 items. Items were designed to elicit yes/no or agree/ disagree answers. There were also prompts allowing for free-text responses. The team wrote a manual of operations and data dictionary that were made available to respondents. Data were collected electronically over the course of one week in October, 2020.

We calculated simple descriptive statistics for numerical data. Two authors (JSL, CM) reviewed the free-text responses and independently identified common themes. We selected representative quotes from each theme for presentation in the results section.

\section{RESULTS}

The total response rate to the audit was $67.4 \%$ (29/43). The majority of centers responding to the audit were in the Northeast region of the United States, were designated as level III or level IV NICUs, and were teaching hospitals (Table 1). Respondents did not differ significantly from non-respondents on these characteristics or the average number of infants eligible for follow-up in the 2019 birth-year cohort.

The majority of programs reported having made changes to their usual practice in response to the pandemic (Table 2). Twenty programs $(69 \%)$ reported altering the capacity of their program to provide care to high-risk infants while nineteen (65.5\%) altered the timing, frequency, or content of medical and developmental assessments. Only one program changed the eligibility criteria for their program due to the pandemic.

Table 1. Sample characteristics.

\begin{tabular}{|c|c|c|c|c|}
\hline & & \multicolumn{2}{|l|}{ Responded } & \multirow[t]{2}{*}{ Total $(N=43)$} \\
\hline & & Yes $(N=29)$ & No $(N=14)$ & \\
\hline \multirow[t]{4}{*}{$\begin{array}{l}\text { Region of } \\
\text { the country }\end{array}$} & $\begin{array}{l}\text { Northeast } \\
\text { (including } \\
\text { Toronto) }\end{array}$ & 14 & 3 & 17 \\
\hline & Midwest & 5 & 4 & 9 \\
\hline & South & 7 & 6 & 13 \\
\hline & West & 3 & 1 & 4 \\
\hline \multirow[t]{3}{*}{ NICU type } & Level II & 1 & 0 & 1 \\
\hline & Level III & 14 & 11 & 25 \\
\hline & Level IV & 13 & 3 & 16 \\
\hline \multirow{2}{*}{$\begin{array}{l}\text { Teaching } \\
\text { hospital }\end{array}$} & Yes & 24 & 12 & 36 \\
\hline & No & 5 & 2 & 7 \\
\hline \multirow{2}{*}{$\begin{array}{l}\text { Eligible } \\
\text { infants for } \\
\text { follow-up } \\
\text { in } 2019\end{array}$} & Mean (SD) & $44(24)$ & $50(24)$ & $46(24)$ \\
\hline & $\begin{array}{l}\text { Median } \\
\text { (Q1, Q3) }\end{array}$ & $42(29,61)$ & $47(27,68)$ & $44(29,62)$ \\
\hline
\end{tabular}

The majority of respondents reported that practice changes due to COVID-19 compromised their ability to make assessments of infants' medical and developmental needs (22; 75.9\%). Fewer $(13 ; 44.8 \%)$ perceived their capacity to assess families' social needs to be diminished. Almost all programs $(25 ; 86.2 \%)$ noted delays in families receiving needed care or services due to the pandemic.

Despite these challenges, many respondents noted some positive changes in their HRIF practices (Fig. 1). Many (17; $58.6 \%)$ agreed that accessibility to their programs and ability to reach families who otherwise might not participate in their programs improved. Eighteen (62.1\%) perceived their programs to be as effective in meeting infant and family needs during as before the pandemic, specifically in following through on new recommendations made during follow-up visits, such as new referrals, and changes to the care plan.

Though only three (13.6\%) programs utilized telemedicine pre-pandemic, the majority $(22 ; 75.9 \%)$ offered telemedicine visits during the pandemic (Table 3 ). Perceived advantages of telemedicine visits included that they were a good substitute for in-person visits during the pandemic, decreased financial and transportation barriers for visits, and increased familycenteredness (Fig. 2). Half (15; 53.6\%) agreed that a benefit of the use of telemedicine is that it allows for virtual 'home visiting'. Perceived drawbacks to using telemedicine included the inability to perform adequate medical and developmental assessments, the cost of technology, and difficulty having multiple team members join a visit.

Respondents had the opportunity to enter free text comments at the end of the audit survey. Four themes emerged from these comments. The first theme related to challenges with performing patient neurological and developmental assessments. As one respondent noted, "Since we are limited in our developmental assessment during the pandemic we are unable to provide appropriate referrals." Another respondent reported that their program adopted the Ages and Stages Questionnaire (ASQ) as a developmental screening tool in lieu of the Bayley Scales of Infant Development (BSID), as this measure cannot be administered via telehealth. They went on to comment, "Though the ASQ provides much information, it cannot replace the full assessment of an [BSID] in-person test."

The second theme concerned difficulty obtaining resources for families and following up recommendations made to families during visits. One respondent said, "We are in closer contact with families, though the types of assessments and therapeutic recommendations we make are limited." Another noted that there were fewer community-based resources and services available to families as a result of the pandemic.

The third theme focused on the challenges clinical providers faced with technology in performing telemedicine visits. According to one respondent, "It is challenging to schedule appointment length of time due to different colleagues/families ability to use technology." Another indicated that "There is significant increase in the amount of preparatory work to enable the session to support the telehealth; such as extra phone calls to the families, introductory of email communication (zoom setup, home setup with appropriate toys)." Respondents also expressed concerns about families lacking the necessary technological resources for telemedicine visits. "A lot of families are working and have school from home too, and that seems to take up their virtual platforms. Some families just don't answer the phone for virtual visits." From another respondent, "Additionally, a good portion of the population that we serve, has little access to the technology (smartphone, wifi, email) or an understanding on its usage for us to offer telehealth." Yet another observed technological barrier that families may face, including having "poor bandwidth, or lose power, or no internet access." 
Table 2. Impact of COVID-19 on high-risk infant follow-through.

\begin{tabular}{|c|c|c|c|}
\hline Program operations & Yes & No & $\%$ Yes \\
\hline \multicolumn{4}{|l|}{ Have you: } \\
\hline Altered the capacity of your program? & 20 & 9 & 69.0 \\
\hline Altered the timing, frequency, or content of assessments? & 19 & 10 & 65.5 \\
\hline \multicolumn{4}{|l|}{ Clinical care } \\
\hline Has your ability to make assessments of infants' medical and developmental needs been compromised? & 22 & 7 & 75.9 \\
\hline \multicolumn{4}{|l|}{$\begin{array}{l}\text { Are you able to follow through on the recommendations you make during your follow-up visits, such as new referrals, } \\
\text { changes to the care plan, etc.? }\end{array}$} \\
\hline Do you perceive delays in needed care or services due to the pandemic? & 25 & 4 & 86.2 \\
\hline \multicolumn{4}{|l|}{ Perceived improvements } \\
\hline \multicolumn{4}{|l|}{ In your opinion, have the changes made to your program improved: } \\
\hline The ability to reach families who otherwise might not participate in your program? & 17 & 12 & 58.6 \\
\hline Equitable allocation of your program resources? & 7 & 22 & 24.1 \\
\hline
\end{tabular}

Perceived Improvments in Follow-Through During COVID-19 Pandemic ( $\mathrm{N}=29)$

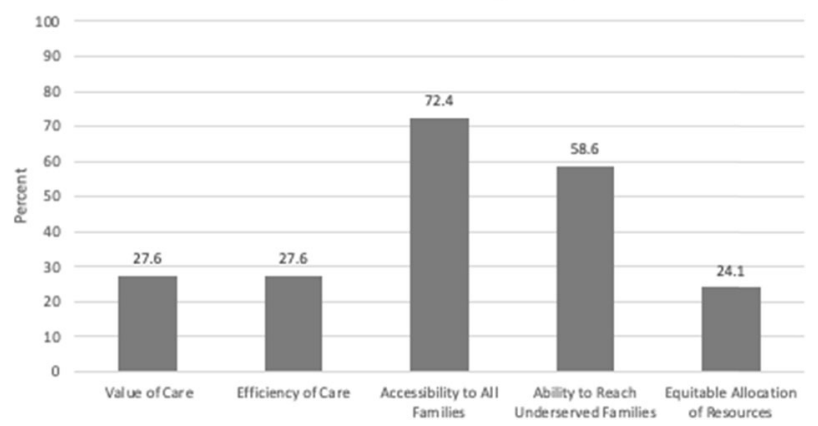

Fig. 1 Perceived enhancements to follow-through care practices in response to COVID: 19 among 29 respondents. The bars represent the proportion of respondents endorsing each attribute of care.

The fourth and final theme related to the impact of telemedicine on increasing program capacity and improving the ability of families to access services. Several respondents noted decreased availability of clinical space for face-to-face visits during the pandemic and parental discomfort with in-person visits due to infection concerns. Despite these limitations, many endorsed improved outreach and communication with families facilitated by telemedicine virtual visits. One respondent found an unexpected benefit of telemedicine visits, in that they made it "[e]asier to engage families who live further from our clinic site who may not otherwise have followed up." Another respondent related an additional unanticipated benefit of telemedicine visits offered during the pandemic,

With telemedicine "home visits" we have been able to see patients that would not have been able to come at all. Although its(sic) not an ideal way to provide care, we were able to make the best judgment based on what we can see and initiate services or mail the family home exercises. We were able to accommodate more patients in the satellite clinics through Telemedicine since we were not having to travel and were able to work from our main location.

\section{DISCUSSION}

Our audit of VON ELBW Infant Follow-up Study Group members found that many high-risk infant follow-up programs adapted rapidly in response to the pandemic. Eighteen centers launched telehealth programs anew during the initial phases of the COVIDrelated shutdown. Many reported having concerns about their ability to perform adequate medical and developmental assessments remotely. Though many observed delays in care due to the pandemic, most felt as effective in meeting patient needs as before the pandemic. Some also expressed concerns about perceived inequities in families' abilities to access to the technology needed to engage in telemedicine visits. Despite these challenges, there was agreement that moving to online platforms increased program capacity and broadened access for families, especially those previously hard to reach.

Infants with medical and developmental complexity require care from multiple sources and in many settings [14]. Access to medical, developmental, and care coordination services is not always a given, particularly for children whose families face social or economic disadvantage, have limited English proficiency, or live in rural areas $[15,16]$. Some high-risk infant follow-up programs have addressed barriers to, and inequities in, access to care through innovative delivery models. The Transition to Home Program at Women and Infant's Hospital uses home visits for high-risk infants and a 24-hour on-call physician line to increase patient access to needed services and reduce rehospitalizations $[17,18]$. A group at Boston Children's Hospital demonstrated the feasibility and acceptability of emailing parents validated health and developmental screening measures to identify risk and need for in-person clinical evaluation for preterm infants [19]. A group in Sweden performed a randomized trial of a home telemedicine intervention for preterm infants after NICU discharge, showing a decrease in clinic and emergency room visits in the first month after discharge [20]. The initial experience described by VON ELBW Follow-up Study Group members lends encouraging early affirmation that digital health approaches can broaden access to follow-up services. Larger-scale utilization and outcomes data will be needed to corroborate this finding, as well as measures of family satisfaction with telemedicine services for highrisk infant follow-up.

Some programs in our network perceived their ability to reach families typically non-compliant with in-person visits improved as 
Table 3. Use of telemedicine for follow-through during the pandemic.

Do you offer telemedicine visits in the assessment or management of your follow-up population?

\begin{tabular}{|c|c|c|}
\hline Yes & $N$ & $\%$ \\
\hline 22 & 29 & 75.9 \\
\hline 3 & 22 & 13.6 \\
\hline 11 & 22 & 50.0 \\
\hline 18 & 22 & 81.8 \\
\hline 7 & 22 & 31.8 \\
\hline 8 & 22 & 36.4 \\
\hline 11 & 22 & 50.0 \\
\hline 2 & 22 & 9.1 \\
\hline
\end{tabular}

For programs offering telemedicine services:

Did you offer telemedicine in the assessment or management of high-risk infants prior to concerns regarding COVID-19?

For which of the following populations andlor criteria do you offer use of telemedicine?

For transition care from the NICU to the follow-up clinic.

For routine visits at standardized age intervals.

To screen for the need of an acute care visit.

To increase the frequency of supportive counseling to the parent or caregiver.

For developmental screening visits.

Other

Do you plan to continue or institute telemedicine in the assessment or management of high-risk infants once your hospital clinics allow in person visits to resume?

What do you perceive are the advantages of the use of telemedicine?

Removes transportation \financial barriers for families.

More family-centered. Allows more family members to join.

Allows multiple sub-specialists to join in visit.

Allows multiple ancillary providers to join in visit.

Allows home visits.

Substitutes for in person visits due to COVID concerns.

Other

\begin{tabular}{lll}
\hline 24 & 28 & 85.7 \\
\hline 13 & 28 & 46.4 \\
\hline 3 & 28 & 10.7 \\
4 & 28 & 14.3 \\
15 & 28 & 53.6 \\
\hline 26 & 28 & 92.9 \\
1 & 28 & 3.6
\end{tabular}

What do you perceive are the barriers or concerns regarding the use of telemedicine?

Availability or cost of technology.

$\begin{array}{lll}13 & 29 & 44.8\end{array}$

Inability to obtain adequate information for assessment.

Coordination of care with team members.

Coordination of care with family members.

Other

a result of practice changes made in response to the pandemic. This perceived improvement in the equitable distribution of follow-up services is a positive consequence of an otherwise challenging situation. Yet, the increased reliance on telemedicine for delivering care may actually lead to a paradoxical worsening of inequities. The availability of reliable broadband internet at the neighborhood level has been associated with online health portal utilization [21]. According to a 2016 survey, $90 \%$ of low-income parents reported having a computer, tablet, or smartphone at home, though half reported having internet connections too slow to complete necessary tasks, 30\% exceeded their data limits at least once a year, and $24 \%$ had internet service shut off due to non-payment [22]. There is also research suggesting that individuals most likely to face health disparities are also most likely to not have access to a computer or internet service [22]. The data from our audit is in agreement with the existing literature, in that many families appear to lack access to reliable internet connections, rely on costly cellular data plans, or lack the equipment to facilitate doctor's visits via telemedicine. We need to ensure that we do not widen inequities in care access or quality in a rush to expand delivery of follow-up care for high-risk infants by telemedicine.

Remote visits using telehealth or other technology have allowed providers to continue offering care while reducing the risk of virus transmission and increased access to needed services, at least for some. However, virtual visits pose challenges to performing assessments of neurologic status, motor function, and other aspects of a child's development. Infant follow-up programs commonly use the Bayley Scales of Infant Development, a standardized test of cognitive, language, and motor development.
The Bayley has not been validated for administration via telemedicine, or with face masks and shields, for that matter. There are validated parent-report screening tools for multiple domains of development in this patient population (e.g., Ages and Stages Questionnaire [23]), though any concerns raised by such parent reports require corroboration with diagnostic tests performed in person. Some problems can be time-sensitive, such as concerns with language or social development, prompting the need for timely diagnosis and intervention. To date, there are few developmental assessment tools that are validated for and easily deployed via telehealth modalities. A group in Australia is testing an approach to performing neurologic assessments, including Prechtl's General Movement Assessment, over telemedicine with families of high-risk infants [24]. The growing interest in--and need for--remote assessments via telemedicine or other modalities makes clear the urgent need for measures that are valid and easy to perform for both clinicians and parents.

With most programs planning to continue offering telemedicine services beyond the pandemic, there is an urgent need to address barriers, develop valid and reliable assessment tools, and set quality standards with focus on equity. First, VON and other networks need to systematically track changes to follow-up rates and the types of follow-up visits being offered (i.e. in-person, virtual, or hybrid). Second, we should work toward identifying risks and supports in the transition from NICU to home and families' capacity to engage with telemedicine services. Lest we widen disparities in care, careful attention must be paid to ensure equitable access to affordable and reliable internet service and we must continue to make available methods of follow-up that are not technology dependent. Third, we need to develop novel 

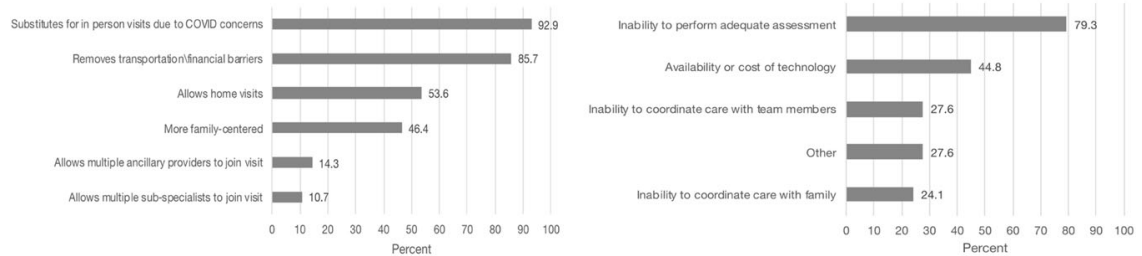

Fig. 2 Perceived advantages of and barriers to telemedicine visits. Bars represent the proportion of respondents endorsing each attribute. The panel on the left denotes perceived benefits and the panel on the right denotes perceived barriers of telemedicine visits.

approaches to performing developmental assessments virtually and procedures for triaging infants for in-person or virtual visits; and we need to ensure infants with identified needs receive indicated therapies and interventions.

Our audit is limited by a small number of respondents and furnishing cross-sectional data from only one point in time. Despite these limitations, the results do provide valuable, and actionable, information about providing follow-up care for highrisk, preterm infants during the COVID-19 pandemic. As programs continue to adapt to the changing needs of patients and their families, providers, and the healthcare delivery system, it is clear that clinical operations, assessment procedures, and quality metrics will also need to evolve. Quality improvement collaboratives like ours are well-positioned to coordinate such work, disseminate key findings, and study the effects of adaptations to local contexts. Regional follow-up collaboratives such as the California Perinatal Care Quality Collaborative and the New England Follow-up Network may serve as incubators for testing change in this arena, providing valuable data on the feasibility, acceptability, and efficacy of novel metrics and practices. It is also clear that high-risk infant follow-up programs have met the challenges posed by the pandemic head-on and have continued to demonstrate their commitment to providing consistent, uninterrupted follow-through services and supports to families after NICU discharge.

\section{REFERENCES}

1. Mehrotra A, Chernew M, Linetsky D, Hatch I, Cutler D. The impact of the COVID-19 pandemic on outpatient visits: changing patterns of care in the newest COVID-19 hot spots. New York, NY; 2020.

2. Birkmeyer JD, Barnato A, Birkmeyer N, Bessler R, Skinner J. The impact of the COVID-19 pandemic on hospital admissions in the United States. Health Aff (Millwood) 2020;39:2010-7.

3. Posfay-Barbe KM, Wagner N, Gauthey M, Moussaoui D, Loevy N, Diana A, et al. COVID-19 in children and the dynamics of infection in families. Pediatrics. 2020;146.

4. Shekerdemian LS, Mahmood NR, Wolfe KK, Riggs BJ, Ross CE, McKiernan CA, et al. Characteristics and outcomes of children with coronavirus disease 2019 (COVID19) infection admitted to US and Canadian pediatric intensive care units. JAMA Pediatr. 2020;174:868-73.

5. Zachariah $\mathrm{P}$, Johnson $\mathrm{CL}$, Halabi KC, Ahn D, Sen Al, Fischer A, et al. Epidemiology, clinical features, and disease severity in patients with coronavirus disease 2019 (COVID-19) in a children's hospital in New York City, New York. JAMA Pediatr. 2020;174:e202430.

6. Rodriguez C, Morris C, Hsu AL. Pediatric care in the age of COVID-19. Pediatr Ann. 2020;49:e403-e4.

7. Houtrow AJ, Okumura MJ, Hilton JF, Rehm RS. Profiling health and health-related services for children with special health care needs with and without disabilities. Acad Pediatr. 2011;11:508-16.

8. Hintz SR, Kendrick DE, Vohr BR, Poole WK, Higgins RD.National Institute of Child Health and Human developement (NICHD) Community supports after surviving extremely low-birth-weight, extremely preterm birth: special outpatient services in early childhood. Arch Pediatr Adolesc Med. 2008;162:748-55.

9. Brown SM, Doom JR, Lechuga-Pena S, Watamura SE, Koppels T. Stress and parenting during the global COVID-19 pandemic. Child Abuse Negl. 2020;110: 104699.

10. Vohr BR, O'Shea M, Wright LL. Longitudinal multicenter follow-up of high-risk infants: why, who, when, and what to assess. Semin Perinatol. 2003;27:333-42.
11. Kuppala VS, Tabangin M, Haberman B, Steichen J, Yolton K. Current state of highrisk infant follow-up care in the United States: results of a national survey of academic follow-up programs. J Perinatol. 2012;32:293-8.

12. Doyle LW, Anderson PJ, Battin M, Bowen JR, Brown N, Callanan C, et al. Long term follow up of high risk children: who, why and how? BMC Pediatr. 2014;14:279.

13. Chanchlani N, Buchanan F, Gill PJ. Addressing the indirect effects of COVID-19 on the health of children and young people. CMAJ 2020;192:E921-E7.

14. Turchi RM, Gatto M, Antonelli R. Children and youth with special healthcare needs: there is no place like (a medical) home. Curr Opin Pediatr. 2007;19:503-8.

15. Miller R, Tumin D, Hayes D Jr., Uffman JC, Raman VT, Tobias JD. Unmet need for care coordination among children with special health care needs. Popul Health Manag. 2019:22:255-61.

16. Toomey SL, Chien AT, Elliott MN, Ratner J, Schuster MA. Disparities in unmet need for care coordination: the national survey of children's health. Pediatrics 2013;131:217-24.

17. Vohr B, McGowan E, Keszler L, Alksninis B, O'Donnell M, Hawes K, et al. Impact of a transition home program on rehospitalization rates of preterm infants. J Pediatr. 2017;181:86-92 e1.

18. Liu Y, McGowan E, Tucker R, Glasgow L, Kluckman M, Vohr B. Transition home plus program reduces medicaid spending and health care use for high-risk infants admitted to the neonatal intensive care unit for 5 or more days. J Pediatr. 2018;200:91-7 e3.

19. Litt JS, Agni M, Jacobi-Polishook T, Melvin P, McCormick MC, Stewart JE, et al. The acceptability and feasibility of emailed parent questionnaires for medical and developmental surveillance after NICU discharge. J Perinatol. 2018;38:392-401.

20. Robinson C, Gund A, Sjoqvist BA, Bry K. Using telemedicine in the care of newborn infants after discharge from a neonatal intensive care unit reduced the need of hospital visits. Acta Paediatr. 2016;105:902-9.

21. Perzynski AT, Roach MJ, Shick S, Callahan B, Gunzler D, Cebul R, et al. Patient portals and broadband internet inequality. J Am Med Inf Assoc. 2017;24:927-32.

22. Katz VS, Gonzalez C, Clark K. Digital inequality and developmental trajectories of low-income, immigrant, and minority children. Pediatrics 2017;140:S132-S6.

23. Kerstjens JM, Nijhuis $A$, Hulzebos CV, van Imhoff DE, van Wassenaer-Leemhuis AG, van Haastert IC, et al. The ages and stages questionnaire and neurodevelopmental impairment in two-year-old preterm-born children. PLoS One. 2015;10: e0133087.

24. Spittle AJ, Rawnsley K. The TEDI Training program: Telehealth for Early Detection and Intervention for Infants with Developmental Disabilities 2020 [The University of Melbourne, Centre of Research Excellence in Newborn Medicine at the Murdoch Children's Research Institute, and Cerebral Palsy Alliance in Australia have developed the TEDI training program: Telehealth for Early Detection and Intervention for Infants with Developmental Disabilities. The TEDI Training program provides online training for clinicians on how telehealth can be used for early detection and intervention for infants with developmental disabilities. The package takes $4 \mathrm{~h}$ to complete and provides interactive content including videos and handouts for families.]. Available from: https://is.gd/TEDIpre.

\section{ACKNOWLEDGEMENTS}

We thank our medical and nursing colleagues who submit data to VON on behalf of infants and their parents. Centers participating in this audit are listed below: Barbara Bush Children's at Maine Medical. Baystate Medical Center. Beth Israel Deaconess Medical Center. Brigham and Women's Hospital. CHOI at OSF St. Francis Medical Center. Children's Minnesota-Minneapolis. Children's Minnesota-St. Paul. Connecticut Children's Medical Center. Connecticut Children's NICU at UCONN Health Center. Dartmouth Hitchcock Medical Center. Driscoll Children's Hospital. Golisano Children's Hospital of Southwest Florida. Kentucky Children's Hospital. Mercy San Juan Medical Center. NHRMC-Betty H. Cameron Women \& Children's Hospital. O.U. Health Sciences Center. Providence Cedars-Sinai Tarzana Medical Center. Rainbow Babies \& Children's Hospital. Saint Barnabas Medical Center. Sunnybrook Health Sciences Centre. Tufts Medical Center. UCSF Benioff Children's Hospital in San Francisco. University Hospital 
San Antonio. University of lowa Children's Hospital. University of Louisville Hospital. University of Vermont Children's Hospital. Women \& Infants Hospital. Yale-New Haven Children's Hospital. Yale-New Haven Children's at Bridgeport Hospital

\section{COMPETING INTERESTS}

The authors declare no competing interests.

\section{ADDITIONAL INFORMATION}

Correspondence and requests for materials should be addressed to J.S.L.

Reprints and permission information is available at http://www.nature.com/ reprints

Publisher's note Springer Nature remains neutral with regard to jurisdictional claims in published maps and institutional affiliations. 